\title{
The Effect of Piezoelectric Stimulation in Patients with Low Fertilization Potential
}

Volkan Baltaci $^{1^{*}}$, Yasemin Aktas ${ }^{2}$, Evrim Unsal ${ }^{2}$, Ozge Uner Ayvaz ${ }^{2}$, Feriba Turhan Eryilmaz $^{2}$, Bilge Sinanoğlu Ekin $^{2}$, Sertaç Şen $^{2}$, Aysun Baltacı $^{2}$

${ }^{1}$ Faculty of Medicine, Department of Medical Genetics, Ufuk University, Ankara, Turkey

${ }^{2}$ Gen Art Woman Health and Reproductive Biotechnology Center, Ankara, Turkey

*Corresponding author: Volkan Baltaci, Faculty of Medicine, Department of Medical Genetics, Ufuk University, Ankara, Turkey, Tel: +90 3125867400 ; E-mail: evrim_unsal@yahoo.com

Received date: February 16, 2014, Accepted date: April 21, 2014, Published date: April 24, 2014

Copyright: (c) 2014 Baltaci V, et al. This is an open-access article distributed under the terms of the Creative Commons Attribution License, which permits unrestricted use, distribution, and reproduction in any medium, provided the original author and source are credited.

\begin{abstract}
Objective: To assess the value of the electrical activation of oocytes in ICSI patients with previous limited fertilization outcomes.

Design: Prospective randomized study.

Settings: Clinical IVF laboratory.

Patient(s): A hundred and seven couples undergoing ICSI with possible low fertilization outcomes.

Intervention(s): TESE, TESA, ICSI with Piezoelectric Activation

Main Outcome Measure(s): Fertilization, clinical pregnancy rates, embryo grades.

Result(s): Patients were subdivided into six study groups. In Group I, testicular elongated spermatids were used and $27.9 \%$ fertilization, $2.3 \%$ clinical pregnancy rates were evaluated. In testicular immotile spermatozoa injected group (Group II), 56.5\% fertilization and 50\% clinical pregnancy rates were obtained. $66.7 \%$ fertilization and $30 \%$ clinical pregnancy rates were achieved in Group III with testicular motile spermatozoa. In Group IV, patients with severe oligozoospermia, $64 \%$ fertilization and $28.6 \%$ clinical pregnancy rates were achieved. Group $\mathrm{V}$ included patients with total immotile spermatozoa and fertilization and clinical pregnancy rates were $50 \%$ and $57.1 \%$ respectively. In patients with history of low fertilization rate (Group VI), $38.7 \%$ fertilization and $19 \%$ clinical pregnancy rates were obtained.
\end{abstract}

Conclusion(s): Piezoelectric stimulation can be used for patients with low fertilization rates and total immotile spermatozoa; as we detected an improvement in the fertilization and clinical pregnancy rates of these patients.

Keywords: Oocyte activation; Piezoelectric; Fertilization rate; Embryo grade; Pregnancy; ICSI
Abbreviations
MPF: Metaphase- Promoting Factor; ICSI: Intra Cytoplasmic Sperm injection; SOAF: Sperm Borne Oocyte-Activating Factors; AOA: Artificial Oocyte Activation; 6-DMAP: Dimethylaminopurine; TESE: Testicular Sperm Extraction; ET: Embryo Transfer; OAT: Oligo Astheno Teratozoospermia; TFF: Total Fertilization Failure; IVF: In Vitro Fertilization
Introduction
Intra Cytoplasmic Sperm Injection (ICSI) has been widely used since 1992 in the era of assisted reproduction [1]. Although this method has become the preferred technique for male infertility, total fertilization failure or low fertilization rates are still the problem that has to be solved. Many studies have focused on this problem and it was

revealed that more than $80 \%$ of unfertilized oocytes were arrested at the metaphase II stage, possibly due to failed oocyte activation [2].

The exact mechanism of oocyte activation is still unclear. Once the spermatozoon binds to receptors on oocyte plasma membrane, either a signal transduction pathway initiates $[3,4]$ or Sperm-borne Oocyteactivating Factors (SOAF) are introduced into the oocyte cytoplasm $[5,6]$. Intracellular $\mathrm{Ca}^{2+}$ increase causesa series of metabolic reactions in oocyte activation process. Higher $\mathrm{Ca}^{2+}$ concentration inactivates Metaphase- Promoting Factor (MPF) that blocks the oocyte cell cycle at the metaphase stage of second meiotic division [7]. Following the inactivation of MPF; oocyte activation occurs and meiosis continues with the anaphase/ telophase transition followed with the exclusion of the second polar body and the exocytosis of cortical granules [8-10].

Several methods have been used for activation of oocytes after ICSI such as electrical activation [11-14], mechanical activation [15,16], and chemical activation such as ethanol [17], $\mathrm{Ca}^{2+}$ ionophores [18-20], strontium [21] and 6-dimethylaminopurine (6-DMAP) [10,22,23]. 
Page 2 of 5

Although oocyte activation can be performed by several methods, the potential toxic effects of chemical activation on different developmental stages of embryos have not been adequately estimated yet. A nonchemical activation method such as electrical stimulation might be used as an alternative against insufficiently tested drugs for activation $[24,25]$.

The aim of this study was to estimate the value of the electrical activation of oocytes in ICSI patients with previously failed or limited fertilization outcomes.

\section{Materials and Methods}

\section{Experimental design}

This study included 107 patients undergoing ICSI treatment at Gen-Art Woman Health and Reproductive Biotechnology Center between January 2006 and May 2009. All the patients were informed about the piezoelectric stimulation procedure and signed an appropriate written consent form. The patients were distributed into six groups. The first group consisted of 43 cycles in which testicular elongated spermatids were used (Group I). In Group II, 6 patients with testicular immotile spermatozoa, in Group III, 10 patients with testicular motile spermatozoa were included. Group IV comprised of 7 patients with severe oligozoospermia, Group V of 7 patients with total immotile spermatozoa and Group VI of 21 patients who had low fertilization rates $(\leq 25 \%)$ in their previous IVF cycles.

\section{Ovarian stimulation and embryo development}

The protocols and procedures for ovarian stimulation and oocyte handling have been published previously [11]. Fertilization was assessed 12-16 hours post microinjection. Normally fertilized oocytes were left for further culture. Embryos were classified according to Veeck's morphological criteria: Grade I embryos had equal-sized blastomeres and no cytoplasmic fragmentation, grade II embryos had blastomeres of equal size and minor cytoplasmic fragmentation covering $10 \%$ of the preembryo surface, grade III embryos had blastomeres of distinctly unequal size and variable fragmentation, grade IV embryos had blastomeres of equal or unequal size and moderate-to-significant cytoplasmic fragmentation covering $>10 \%$ of the pre-embryo surface, and grade $\mathrm{V}$ embryos had few blastomeres of any size and severe fragmentation covering $50 \%$ of the preembryo surface. None of the embryos were classified as grade $\mathrm{V}$ in this study.

All embryos were transferred on the third day of culture, using transabdominal sonographic guidance. Biochemical pregnancy was established when serum $\beta$-HCG was found $>20$ IU/L on the 12th day after embryo transfer, and clinical pregnancy was defined as the presence of a gestational sac on ultrasound examination at 6 week's gestation.

\section{Semen preparation}

For the preparation of ejaculated semen, gradient or swim-up, techniques were performed regarding to sperm parameters [26-28]. Testicular Sperm Extraction (TESE) procedure was performed under local anesthesia by widely opening the testis in an equatorial plane. The collected tissue was mechanically dispersed in a petri dish (Falcon Plastics, Becton- Dickinson) with an insulin syringe. All the elongated spermatids, immotile and motile spermatozoa were collected directly from the pool of testicular tissue samples or testicular sperm aspirates

\section{Piezoelectric stimulation of injected oocytes}

Piezoelectric stimulation was applied 20 minutes following microinjection with a BTX Electro-cell manipulator (BTX, San Diego, CA) at room temperature with a chamber with two stainless steel electrodes $0.5 \mathrm{~mm}$ apart, filled with activation buffer $(\mathrm{pH} \quad 1 / 4 \quad 7.0)$ including Mannitol (0.3 M), $\mathrm{MgSO}_{4}(0.1 \mathrm{mM}), \mathrm{CaCl}_{2}(0.1 \mathrm{mM})$, HSA $(0.05 \mathrm{mg} / \mathrm{mL})$, and HEPES $(0.5 \mathrm{mM})$. Injected oocytes were activated with a single pulse of $1.5 \mathrm{kV} / \mathrm{cm}$ DC for $100 \mathrm{msec}$. Stimulated oocytes were immediately transferred back to culture medium G1 (Vitro life; IVF Science) droplets in a humidified atmosphere of $6 \% \mathrm{CO}_{2}$ at $37^{\circ} \mathrm{C}$.

\section{Statistical analysis}

Data analysis was performed using Statistical Package for Social Sciences (SPSS) version 11.5 software (SPSS Inc., , , ).

\section{Results}

A total of 498 embryos were obtained after piezoelectric stimulation from 107 patients. The effect of electro activation on fertilization and clinical pregnancy rates are described in Table 1. In Group I, 27.9\% fertilization and $2.3 \%$ clinical pregnancy rates were achieved. In Group II, the fertilization and clinical pregnancy rates were 56.5\% and 50\% respectively whereas the rates were $66.7 \%$ and $30 \%$ in Group III. Piezoelectric stimulation was also applied to patients with severe oligozoospermia in order to increase the fertilization rate and $64 \%$ fertilization and $28.6 \%$ clinical pregnancy rates were achieved (Group IV). In Group V, fertilization rate was $50 \%$ and clinical pregnancy rate was $57.1 \%$. In patients with a history of low fertilization rate (Group VI), $38.7 \%$ fertilization and $19 \%$ clinical pregnancy rates were obtained.

\begin{tabular}{|l|l|l|l|}
\hline \multicolumn{2}{|l|}{ Groups } & Fertilization Rate\% & $\begin{array}{l}\text { Clinical Pregnancy } \\
\text { Rate } \%\end{array}$ \\
\hline Group I $(n=43)$ & Testicular Elongated & 27.9 & 2.3 \\
\hline Group II $(n=6)$ & TESE-Immotile & 56.5 & 50.0 \\
\hline Group III $(n=10)$ & TESE-Motile & 66.7 & 30.0 \\
\hline Group IV $(n=7)$ & Severe Oligozoospermia & 64.0 & 28.6 \\
\hline Group V $(n=7)$ & Total Immotile & 50.0 & 57.1 \\
\hline
\end{tabular}




\begin{tabular}{|l|l|l|l|}
\hline Group VI $(\mathrm{n}=21)$ & Low Fertilization Rate & 38.7 & 19.0 \\
\hline
\end{tabular}

Table 1: The Effect of Piezoelectric Stimulation on Fertilization and Clinical Pregnancy Rates among Six Study Groups

The grades of embryos were also evaluated in each group to see whether electro stimulation had apparent effects on embryo morphology or not. The grades of embryos were also classified among 6 groups and individually figured out in Table 2 .

\begin{tabular}{|l|l|l|l|}
\hline \multicolumn{2}{|l|}{ groups } & Grade 1-2 & Grade 3-4 \\
\hline Group I $(n=116)$ & Testicular Elongated & $37.1 \%$ & $62.9 \%$ \\
\hline Group II $(n=65)$ & TESE-Immotile & $41.5 \%$ & $58.5 \%$ \\
\hline Group III $(n=115)$ & TESE-Motile & $47.0 \%$ & $53.0 \%$ \\
\hline Group IV $(n=50)$ & Severe Oligozoospermia & $64.0 \%$ & $36.0 \%$ \\
\hline Group V $(n=56)$ & Total Immotile & $71.4 \%$ & $28.6 \%$ \\
\hline Group VI $(n=96)$ & Low Fertilization Rate & $39.6 \%$ & $60.4 \%$ \\
\hline
\end{tabular}

Table 2: Quality of Embryos in Each Study Group Following Piezoelectric Stimulation

\section{Discussion}

Fertilization failures occur in $2 \%-3 \%$ of ICSI cycles $[29,30]$. Cytological analysis estimate that more than half of the fertilization failures of human oocytes after ICSI are due to insufficient oocyte activation [31]. Nowadays, recurrent failed fertilization cases can only be solved by using assisted oocyte activation [32]. The most widely adopted agents for human oocytes include $\mathrm{Ca}^{2+-}$ ionophore and ionomycin or electrical stimuli [33].

Oocyte activation method has been used as an efficient treatment option in cases of complete fertilization failure and low fertilization outcomes [19,24,34-40].

Cytoplasmic aspiration in conventional ICSI procedure may not always be sufficient for oocyte activation [16,23]. There are several studies focusing on different artificial oocyte activation (AOA) techniques. According to some of the previously published data, artificially activated oocytes (either calcium ionophore or electrically) develop similarly to fertilized oocytes [41-44].

Tejera et al. [19], showed that activation of oocytes with calcium ionophore increased the fertilization rates from $35.7 \%$ to $55.6 \%$ and they achieved a successful pregnancy and childbirth [19]. On the contrary Borges et al. [18] reported that AOA with Calcium ionophore did not improve ICSI outcomes with epidydimal or testicular spermatozoa. Also Kahraman previously reported that oocyte activation prior to ICSI did not lead up to better fertilization rates [45].

The knowledge about the potential cytotoxic, teratogenic and mutagenic effects of assisted activation techniques on oocytes and embryos in IVF is inadequate $[26,46]$. While designing our studies, we preferred the nonchemical activation method such as electrical stimulation $[12,24,26]$ as chemical activation was shown to induce parthenogenetic development of oocytes [47]. On the other hand about $70 \%-80 \%$ of unfertilized oocytes after ICSI responded to electro activation and formed two pronuclei $[24,36]$.

In the recent study we aimed to evaluate the effect of piezoelectric stimulation in patients with possible low fertilization outcomes. In the beginning we decided to evaluate elongated spermatid injected oocytes and observed a fertilization rate of $27.9 \%$ whereas various studies have reported $24 \%[48,49]$ and $71 \%$ [50]. Although we had piezoelectrically stimulated the oocytes of patients with elongated spermatids; our data is still similar to non-activated studies. Although we achieved one successful clinical and one biochemical pregnancy, the results were still unsatisfactory. Furthermore, in the study of Kumagai et al., 15\% of pregnancy rate was reported with electro activation of spermatid injection [51].

Testicular immotile spermatozoa injection combined with piezoelectric stimulation, the fertilization rates were observed as $56.5 \%$ while the clinical pregnancy rates were $50 \%$. In case of using testicular motile spermatozoa; fertilization rates were $66.7 \%$ and clinical pregnancy rates were $30 \%$. Konc et al. [52] have evaluated the nonactivated TESE-ICSI-ET cycles and found a fertilization rate of $68 \%$ and $60 \%$ and a pregnancy rate of $33 \%$ and $29 \%$ with TESE motile spermatozoon and TESE immotile spermatozoon respectively. When we compare our results with this study, we observed no significant difference in fertilization and pregnancy rates in testicular motile spermatozoon injected patients. However the results of TESE immotile group indicated an impressive effect of piezoelectric activation on pregnancy rates.

Implantation period, postimplantational development, fetal morphology [53] and weight variations in offspring could be affected via the differences in the calcium oscillation [54]. The exact mechanism by which intracellular calcium influences embryonic development remain subject to debate, [18] Borges et al. [18] have demonstrated higher pregnancy rates in artificial oocyte activation by calcium ionophore A23187 and also indicated that more patients are needed to be evaluated [18]. In our study, it was an unexpected result for us to find that electro activation had affected only pregnancy rate but not fertilization rate in TESE immotile group. So we concluded that it would be appropriate to increase the number of patients in order to obtain statistically significant data.

In a previous study of Mansour et al., the fertilization rates were $68 \%$ in electro activated patients with severe 
oligoasthenoteratozoospermia (OAT) and azoospermia whereas the $60 \%$ in control group [12]. We also intended to evaluate the effects of piezoelectric stimulation on severe oligozoospermia patients' IVF cycles and couldn't observe any significant difference as our fertilization rate was $64 \%$.

On the other hand we obtained sufficient results in total immotile group (Group V); a pregnancy rate of $57.1 \%$ and good quality embryo rate of $71.4 \%$. A fertilization rate of $44 \%$ was reported in Westlander et al. [55] study and 2 of 6 embryos were found to be good quality. As Nijset et al. [56], obtained low quality embryos with total immotile spermatozoa, our results referred the positive effect of piezoelectric stimulation on embryo grades and clinical pregnancy rates of total immotile patients. It has always been questioned whether to use testicular or seminal sperm for ICSI in total immotile patients. Westlander et al. [55], preferred to use testicular immotile spermatozoa instead of seminal immotile as DNA damage increases in ejaculated semen [55]. In contrary to this, our results demonstrated that higher pregnancy rates and better quality of embryos were obtained by using seminal immotile sperm for microinjection.

Hee-Jun Chi et al. demonstrated that the fertilization rate was increased from $25 \%$ to $80 \%$ after calcium ionophore activation in a normozoospermic patient with a history of low fertilization rate and obtained a successful pregnancy and a twin born following the activation [43]. Our results have not suggested such a significant increase in fertilization rates but still, piezoelectric could be referred as a sufficient activation method in patients with low fertilization histories.

In our previous study; we obtained very encouraging results of piezoelectric combined ICSI in Total Fertilization Failure (TFF) patients and reported apregnancy rate of $12.8 \%$ [11]. Yanagida et al. [24] reported that ICSI followed by electrical oocyte activation resulted in the delivery of healthy twins for a couple with previously failed fertilization [24]. Mansour et al. [12], also recommended that the patients should be electro activated if there was a failure/limitation in previous cycles or the patient was severe OAT, azoospermic or has total immotile spermatozoa [12].

In studies related with total or limited failure of fertilization, all the activation procedures are performed on oocytes. On the other hand, it has been estimated that sperm soluble factors also plays a role in oocyte activation. Yanagida et al. [57] compared the fertilization, cleavage and pregnancy rates among three types of sperm immobilization (pipetting, squeezing and piezo application) techniques and concluded that the piezo activation method was the most efficient one. In order to use artificial activation techniques as a part of the conventional IVF procedures; the effects of sperm soluble factors could also be taken into consideration in multifactorial oocyte activation studies.

As a conclusion; our results agree with the usage of piezoelectric stimulation for patients with low fertilization rates and total immotile spermatozoa; as we detected an improvement in the fertilization and clinical pregnancy rates of these patients. But further research is still needed with an increased number of patients in order to evaluate the clinical effectiveness and safety of this method.

\section{References}

1. Palermo G, Joris H, Devroey P, Van Steirteghem AC (1992) Pregnancies after intracytoplasmic injection of single spermatozoon into an oocyte. Lancet 340: 17-18.
2. Tesarik J, Sousa M, Testart J (1994) Human oocyte activation after intracytoplasmic sperm injection. Hum Reprod 9: 511-518.

3. Miyazaki S, Shirakawa H, Nakada K, Honda Y (1993) Essential role of the inositol 1,4,5-triphosphate receptor/ Ca2+ release channel in Ca2+ waves and $\mathrm{Ca} 2+$ oscillations at fertilization of mammalian eggs. DevBiol 158: 62-78.

4. Schultz RM, Kopf GS (1995) Molecular basis of mammalian egg activation. Curr Top Dev Biol 30: 21-62.

5. Swann K, Lai FA (1997) A novel signalling mechanism for generating Ca2+ oscillations at fertilization in mammals. Bioessays 19: 371-378.

6. Parrington J, Lai FA, Swann K (1998) A novel protein for Ca2+ signaling at fertilization. Curr Top Dev Biol 39: 215-243.

7. Sagata N (1996) Meiotic metaphase arrest in animal oocytes: its mechanisms and biological significance. Trends Cell Biol 6: 22-28.

8. Liu L1, Ju JC, Yang X (1998) Parthenogenetic development and protein patterns of newly matured bovine oocytes after chemical activation. Mol Reprod Dev 49: 298-307.

9. Liu L, Ju JC, Yang X (1998) Differential inactivation of maturationpromoting factor and mitogen-activated protein kinase following parthenogenetic activation of bovine oocytes. BiolReprod 59: 537-545.

10. Devito LG, Fernandes CB, Blanco ID, Tsuribe PM, Landim-Alvarenga FC (2010) Use of a Piezo drill for intracytoplasmic sperm injection into cattle oocytes activated with ionomycin associated with roscovitine. Reprod Domest Anim 45: 654-658.

11. Baltaci V, Ayvaz OU, Unsal E, AktaÅŸ Y, Baltaci A, et al. (2010) The effectiveness of intracytoplasmic sperm injection combined with piezoelectric stimulation in infertile couples with total fertilization failure. Fertil Steril 94: 900-904.

12. Mansour R, Fahmy I, Tawab NA, Kamal A, El-Demery Y, et al. (2009) Electrical activation of oocytes after intracytoplasmic sperm injection: a controlled randomized study. Fertil Steril 91: 133-139.

13. Haigo K, Otsuka M, Egashira A, YoshiokaN, KuramotoT (2006) Pregnancy and delivery following ICSI with electrical activation in a case of globozoospermia. FertilSteril 86: S358.

14. Zimmermann U, Vienken J (1982) Electric field-induced cell-to-cell fusion. J Membr Biol 67: 165-182.

15. Van Blerkom J, Davis P, Alexander S (2003) Inner mitochondrial membrane potential (DeltaPsim), cytoplasmic ATP content and free Ca2+ levels in metaphase II mouse oocytes. Hum Reprod 18: 2429-2440.

16. Ebner T, Moser M, Sommergruber M, Jesacher K, Tews G (2004) Complete oocyte activation failure after ICSI can be overcome by a modified injection technique. Hum Reprod 19: 1837-1841.

17. Ilyin V, Parker I (1992) Effects of alcohols on responses evoked by inositol trisphosphate in Xenopus oocytes. J Physiol 448: 339-354.

18. Borges E Jr, de Almeida Ferreira Braga DP, de Sousa Bonetti TC, Iaconelli A Jr, Franco JG Jr (2009) Artificial oocyte activation with calcium ionophore A23187 in intracytoplasmic sperm injection cycles using surgically retrieved spermatozoa. FertilSteril 92:131-136.

19. Tejera A, Mollá M, Muriel L, Remohí J, Pellicer A, et al. (2008) Successful pregnancy and childbirth after intracytoplasmic sperm injection with calcium ionophore oocyte activation in a globozoospermic patient. Fertil Steril 90: 1202 .

20. Nakada K, Mizuno J (1998) Intracellular calcium responses in bovine oocytes induced by spermatozoa and by reagents. Theriogenology 50 : 269-282.

21. Yamazaki W, Ferreira CR, Me SC (2003) Normal offspring produced by somatic cell nuclear transfer using strontium and ionomycin as activating agents. Theriogenology 59: 293.

22. Galli C, Duchi R, Moor RM, Lazzari G (1999) Mammalian leukocytes contain all the genetic information necessary for the development of a new individual. Cloning 1: 161-170.

23. Nasr-EsfahaniMH, Reza Deemeh M, Tavalaee M (2010) Artificial oocyte activation and intracytoplasmic sperm injection. FertilSteril 94: 520-526. 
24. Yanagida K, Katayose H, Yazawa H, Kimura Y, Sato A, et al. (1999) Successful fertilization and pregnancy following ICSI and electrical oocyte activation. Hum Reprod 14: 1307-1311.

25. Egashira A, Murakami M, Haigo K, Horiuchi T, Kuramoto T (2009) A successful pregnancy and live birth after intracytoplasmic sperm injection with globozoospermic sperm and electrical oocyte activation. Fertil Steril 92: 2037.

26. Morshedi M, Duran HE, Taylor S, Oehninger S (2003) Efficacy and pregnancy outcome of two methods of semen preparation for intrauterine insemination: a prospective randomized study. Fertil Steril 79 Suppl 3: 1625-1632.

27. Jakab A, Sakkas D, Delpiano E, Cayli S, Kovanci E, et al. (2005) Intracytoplasmic sperm injection: a novel selection method for sperm with normal frequency of chromosomal aneuploidies. Fertil Steril 84: 1665-1673.

28. World Health Organization (1999). WHO laboratory manual for the examination of human semen and sperm-cervical mucus interaction. Cambridge, UK: Cambridge University Press.

29. Mahutte NG, Arici A (2003) Failed fertilization: is it predictable? Curr Opin Obstet Gynecol 15: 211-218.

30. Heindryckx B, Van der Elst J, De Sutter P, Dhont M (2005) Treatment option for sperm- or oocyte-related fertilization failure: assisted oocyte activation following diagnostic heterologous ICSI. Hum Reprod 20: 2237-2241.

31. Rawe VY, Olmedo SB, Nodar FN, Doncel GD, Acosta AA, et al. (2000) Cytoskeletal organization defects and abortive activation in human oocytes after IVF and ICSI failure. Mol Hum Reprod 6: 510-516.

32. Neri QV, Lee B, Rosenwaks Z, Machaca K, Palermo GD (2014) Understanding fertilization through intracytoplasmic sperm injection (ICSI). Cell Calcium 55: 24-37.

33. Heindryckx B, De Gheselle S, Gerris J, Dhont M, De Sutter P (2008) Efficiency of assisted oocyte activation as a solution for failed intracytoplasmic sperm injection. Reprod Biomed Online 17: 662-668.

34. Yanagida K (2004) Complete fertilization failure in ICSI. Hum Cell 17: 187-193.

35. Rybouchkin AV, Van der Straeten F, Quatacker J, De Sutter P, Dhont M (1997) Fertilization and pregnancy after assisted oocyte activation and intracytoplasmic sperm injection in a case of round-headed sperm associated with deficient oocyte activation capacity. Fertil Steril 68: 1144-1147.

36. Kim ST, Cha YB, Park JM, Gye MC (2001) Successful pregnancy and delivery from frozen-thawed embryos after intracytoplasmic sperm injection using round-headed spermatozoa and assisted oocyte activation in a globozoospermic patient with mosaic Down syndrome. Fertil Steril 75: 445-447.

37. Eldar-Geva T, Brooks B, Margalioth EJ, Zylber-Haran E, Gal M, et al. (2003) Successful pregnancy and delivery after calcium ionophore oocyte activation in a normozoospermic patient with previous repeated failed fertilization after intracytoplasmic sperm injection. FertilSteril 79: 16561658.

38. Chi HJ, Koo JJ, Song SJ, Lee JY, Chang SS (2004) Successful fertilization and pregnancy after intracytoplasmic sperm injection and oocyte activation with calcium ionophore in a normozoospermic patient with extremely low fertilization rates in intracytoplasmic sperm injection cycles. FertilSteril 82: 475-477.

39. Murase Y, Araki Y, Mizuno S, Kawaguchi C, Naito M, et al. (2004) Pregnancy following chemical activation of oocytes in a couple with repeated failure of fertilization using ICSI: case report. Hum Reprod 19: 1604-1607.

40. Vanderzwalmen P, Zech H, Birkenfeld A, Yemini M, Bertin G, et al. (1997) Intracytoplasmic injection of spermatids retrieved from testicular tissue: influence of testicular pathology, type of selected spermatids and oocyte activation. Hum Reprod 12: 1203-1213.
41. Parrington J, Swann K, Shevchenko VI, Sesay AK, Lai FA (1996) Calcium oscillations in mammalian eggs triggered by a soluble sperm protein. Nature 79: 364-368.

42. Kim ST, Cha YB, Park JM, Gye MC (2001) Successful pregnancy and delivery from frozen-thawed embryos after intracytoplasmic sperm injection using round-headed spermatozoa and assisted oocyte activation in a globozoospermic patient with mosaic Down syndrome. FertilSteril 75: 445-447.

43. Chi HJ, KooJJ, SongSJ, LeeJY, Chang SS (2004) Successful fertilization and pregnancy after intracytoplasmic sperm injection and oocyte activation with calcium ionophore in a normozoospermic patient with extremely low fertilization rates in intracytoplasmic sperm injection cycles. FertilSteril 82: 475-477.

44. van der Westerlaken L, Helmerhorst F, Dieben S, Naaktgeboren N (2005) Intracytoplasmic sperm injection as a treatment for unexplained total fertilization failure or low fertilization after conventional in vitro fertilization. Fertil Steril 83: 612-617.

45. Kahraman S, Polat G, Sözen E, Akarsu C, Özbicer T, et al. (1999) Oocyte activation before intracytoplasmic sperm injectiondoes not improve the fertilization and embryo quality. European Society of Human Reproduction Andrology and Embryology, 15th Annual Meeting.

46. Tesarik J, Rienzi L, Ubaldi F, Mendoza C, Greco E (2002) Use of a modified intracytoplasmic sperm injection technique to overcome sperm-borne and oocyte-borne oocyte activation failures. Fertil Steril 78: 619-624.

47. Paffoni A, Brevini TA, Somigliana E, Restelli L, Gandolfi F, et al. (2007) In vitro development of human oocytes after parthenogenetic activation or intracytoplasmic sperm injection. Fertil Steril 87: 77-82.

48. Fishel S, Green S, Hunter A, Lisi F, Rinaldi L, et al. (1997) Human fertilization with round and elongated spermatids. Hum Reprod 12: 336-340.

49. Mansour RT, Fahmy IM, Taha AK, Tawab NA, Serour GI, et al. (2003) Intracytoplasmic spermatid injection can result in the delivery of normal offspring. J Androl 24: 757-764.

50. Kahraman S, Polat G, Samli M, Sözen E, Ozgün OD, et al. (1998) Multiple pregnancies obtained by testicular spermatid injection in combination with intracytoplasmic sperm injection. Hum Reprod 13: 104-110.

51. Kumagai A, Tanaka C, Nishinaka Y, Nakajo H, Uto K (2006) Three successful pregnancies and deliveries after oocyte activation by strontium chloride (SRCL2) in six patients of low fertilization rate. FertilSteril 86: S156-S157.

52. Konc J, Kanyó K, Cseh S (2008) The effect of condition/state of testicular spermatozoa injected to the outcome of TESE-ICSI-ET cycles. Eur J Obstet Gynecol Reprod Biol 141: 39-43.

53. Ozil JP, Huneau D (2001) Activation of rabbit oocytes: the impact of the $\mathrm{Ca} 2+$ signal regime on development. Development 128: 917-928.

54. Ozil JP, Banrezes B, Tóth S, Pan H, Schultz RM (2006) Ca2+ oscillatory pattern in fertilized mouse eggs affects gene expression and development to term. Dev Biol 300: 534-544.

55. Westlander G, Barry M, Petrucco O, Norman R (2003) Different fertilization rates between immotile testicular spermatozoa and immotile ejaculated spermatozoa for ICSI in men with Kartagener's Syndrome: case report. Hum Reprod 18: 1286-1288.

56. Nijs M, Vanderzwalmen P, Vandamme B, Segal-Bertin G, Lejeune B, et al. (1996) Fertilizing ability of immotile spermatozoa after intracytoplasmic sperm injection. Hum Reprod 11: 2180-2185.

57. Yanagida K, Katayose H, Hirata S, Yazawa H, Hayashi S, et al. (2001) Influence of sperm immobilization on onset of $\mathrm{Ca}(2+)$ oscillations after ICSI. Hum Reprod 16: 148-152. 